\title{
Effects of synthetic colloid and crystalloid solutions on hemorheology in vitro and in hemorrhagic shock
}

Gan Chen, Jingxiang Zhao, Penglong Li, Xuemei Kan, Guoxing You, Ying Wang, Yujing Yin, Xin Luo, Yuhua Zhang, Lian Zhao ${ }^{*}$ and Hong Zhou*

\begin{abstract}
Background: Plasma expanders are commonly used in the management of critically ill patients, which may exhibit altered hemorheology. We evaluated the effects of various synthetic colloids and Lactated Ringer's (LR) solution on hemorheological parameters in vitro and in a rodent hemorrhagic shock model.

Methods: For the in vitro experiments, rat blood was incubated with hydroxyethyl starch (HES) 130/0.4, HES 200/0.5, succinylated gelatine (GEL), or LR at various ratios. The control consisted of blood without dilution. The hemorheological parameters were measured after a 15-min incubation. For the in vivo study, rats were subjected to a severe volume-controlled hemorrhage and were resuscitated using a colloid solution (HES 130/0.4, HES 200/0.5, or GEL) or LR. The hemorheological parameters were measured $2 \mathrm{~h}$ after resuscitation.

Results: The GEL significantly elevated the plasma viscosity compared to the other groups. In the in vitro study, GEL and LR accelerated the erythrocyte aggregation. There was no significant difference between HES 130/0.4, HES 200/0.5, and control groups regarding the aggregation amplitude and index. In the in vivo study, the aggregation amplitude increased significantly in the GEL group compared to the HES 130/0.4, HES 200/0.5, LR, and sham groups. There was no significant difference between the groups with respect to the elongation index in vivo.

Conclusions: Hydroxyethyl starch did not change the erythrocyte aggregation compared to the control. GEL significantly accelerates the erythrocyte aggregation and elevates the plasma viscosity compared to hydroxyethyl starch. The in vitro hemorheological measurements most likely provide hints for the in vivo study.
\end{abstract}

Keywords: Plasma expander, Synthetic colloids, Hemorrhagic shock, Plasma viscosity, Erythrocyte aggregation

\section{Background}

The administration of plasma expanders is crucial for managing critically ill patients across a range of clinical conditions, including major surgery, hemorrhagic shock (HS), and trauma [1]. There is growing evidence that plasma expanders, which are often given in large volumes during fluid therapy, are associated with altered hemorheological parameters, which play an important role in modulating microcirculatory and tissue oxygenation $[2,3]$. Previous studies have demonstrated the effects of various fluids on hemorheology [4-7]. Dextran increased the plasma viscosity and induced erythrocyte aggregation

\footnotetext{
* Correspondence: zhaolian@bmi.ac.cn; zhouhtt1966@163.com Institute of Transfusion Medicine, Academy of Military Medical Sciences, No. 27th Taiping Road, HaiDian, Beijing, China
}

by forming dextran bridges between the cells $[8,9]$. Hydroxyethyl starch (HES) 450/0.7 infusion elevated blood viscosity and erythrocyte aggregation while decreasing the erythrocyte deformability [6]. Gelatin was also found to increase erythrocyte aggregation [10]. However, direct comparisons of the hemorheological effects between plasma expanders, such as succinylated gelatin, HES 130/0.4, HES 200/0.5, and Lactated Ringer's (LR) solution, are lacking. Among the hemorheological parameters, erythrocyte aggregation may be associated with tissue perfusion $[11,12]$. However, the effects of HES on erythrocyte aggregation remain controversial [4]. HES 200/0.5 and HES 130/0.4 were indicated to induce erythrocyte aggregation in both in vitro and in vivo studies $[6,13,14]$. However, other studies have shown 
that erythrocyte aggregation was unchanged in the presence of HES 200 compared to that observed in the control group $[4,15]$.

The rheological measurements can be investigated in vitro and in vivo. In vitro techniques could permit screening of the rheological properties of different plasma expanders, such as hemoglobin-based oxygen carriers, by mixing erythrocytes and expanders in different ratios [16]. Moreover, the in vitro rheological effects of different solutions can be examined in normal and septic blood to predict further compromise in vivo [6]. In vitro rheological measurements show advantage in saving time and animal models. However, the in vitro rheological effects may differ significantly from the in vivo effects. The extent to which in vitro findings for hemorheological parameters are of relevancy for the in vivo situation needs to be further investigated.

Thus, to evaluate the effects of synthetic colloids and LR solution on hemorheology and the relationship between in vitro and in vivo rheological measurements, we compared HES 130/0.4, HES 200/0.5, succinylated gelatin, and LR to demonstrate their relative effects on hemorheology in vitro. Next, we verified the in vitro results using a rodent HS model in vivo. The hemorheological parameters include plasma viscosity, erythrocyte aggregation, and erythrocyte deformability.

\section{Methods}

\section{Animal preparation}

This study was approved by the ethics committee of the Institute of Transfusion Medicine, Academy of Military Medical Sciences. All efforts were made to minimize the number of animals used and their suffering. Fourty-eight male Wistar rats weighing 210-250 g (Vital River Laboratories, Beijing, China) were included in the study; 39 rats were used for the in vivo experiment. The remaining rats were used for the in vitro experiment. Animals were used after a minimum 5- to 7-day acclimation period.

The rats were anesthetized using sodium pentobarbital $(50 \mathrm{mg} / \mathrm{kg})$, which were injected intraperitoneally. Rats were allowed to breathe room air spontaneously in a supine position for the duration of the experiment. Body temperature was maintained at $37^{\circ} \mathrm{C} \pm 0.1^{\circ} \mathrm{C}$ with the aid of a heating pad (Softron Beijing, Inc., TMS-201, China). The right femoral artery and vein were isolated with minimal dissection and catheterized using polyethylene catheters (PE-50) containing heparinized saline. Rats were heparinized with $400 \mathrm{U} / \mathrm{kg}$ heparin (IV). Supplementary doses of pentobarbital were administered when necessary.

\section{Blood sampling and treatment for the in vitro study}

Fresh blood samples were obtained from the right femoral arterial catheter using heparin-treated tubes $(15 \mathrm{U} / \mathrm{mL})$. The rat blood was centrifuged for $5 \mathrm{~min}$ at $1,500 \times \mathrm{g}$ and adjusted to a hematocrit of $40 \%$ by adding or removing autologous plasma. To represent the clinically relevant colloid plasma peak levels reported in the literatures $[9,17,18]$, the well-distributed erythrocyte samples were subsequently diluted with 6\% HES 130/0.4 (HES 130) (Voluven', Fresenius-Kabi, Bad Homburg, Germany), 6\% HES 200/0.5 (HES 200) (HAES-steril ${ }^{\circ}$, Fresenius-Kabi, Beijing, China), $4 \%$ succinylated gelatin (GEL, $30 \mathrm{kDa}$ ) (Gelofusine ${ }^{\circ}$ B. Braun, Shenyang, China), or LR (Juneng, Siping, China); the volume ratios of blood with the test solutions are 5:1 and 3:1. The control group consisted of the erythrocyte suspension without dilution.

All samples were incubated for $15 \mathrm{~min}$ at $37^{\circ} \mathrm{C}$, and then, blood samples were adjusted to a hematocrit of $40 \%$ before measuring the hemorheological parameters. The plasma was obtained using centrifugation to detect the viscosity. All rheological measurements were carried out at a temperature of $37^{\circ} \mathrm{C}$, which are in accordance with the international guidelines for the measurement of hemorheological parameters [19].

\section{Hemorrhagic shock/resuscitation protocol for the in vivo study}

A rat HS model was prepared as described previously [20]. After surgical preparation and $10 \mathrm{~min}$ of stabilization, a volume-controlled hemorrhage of $18 \mathrm{~mL} / \mathrm{kg}$ was performed using pumps (Softron Beijing, Inc., Beijing, China) for 30 min through the right femoral arterial catheter. Rats were then subjected to a slower hemorrhage of $12-15 \mathrm{~mL} / \mathrm{kg}$ for $35 \mathrm{~min}$. Due to the individual differences of animals, the blood withdrawal rate changed with a certain range. The animals with a base excess of -9 to $-12 \mathrm{mmol} / \mathrm{L}$ were resuscitated after blood withdrawal via the femoral venous catheter. The results of arterial blood gas analysis have been published in our previous study [20].

After the volume-controlled hemorrhage was induced, the animals were randomly assigned to one of five groups. The sham group underwent all instrumentation procedures without blood collection $(n=7)$. The other four groups each received a different colloid solution (HES 130, HES 200, GEL) or LR ( $n=8$ /group). The volume of resuscitation for the colloid solutions was equal to that of the blood withdrawal and three times that of the blood withdrawal for LR; all the infusions were performed using a pump-driven constant infusion at a rate of 0.33 $\mathrm{mL} / \mathrm{min}$. After $2 \mathrm{~h}$ had elapsed following resuscitation, blood samples were collected from the femoral artery with heparin-treated tubes for hemorheological evaluation $(15 \mathrm{U} / \mathrm{mL})$. The animals were then euthanized by exsanguination under anesthesia.

\section{Hemorheological evaluation}

Hemorheology was assessed through the plasma viscosity, erythrocyte deformability (elongation index (EI)), 
erythrocyte aggregation index (AI), and aggregation amplitude (AMP).

The plasma viscosity was measured using a capillary viscometer at $37^{\circ} \mathrm{C}$ (LBY-N6B, Precil Company, Beijing, China). The measurement of viscosity was based on analyzing the capillary pressure-driven movement of plasma whose mean velocity and, therefore, shear rate varies with time.

EI was measured at shear rates of $100,400,600,800$, and $1,000 \mathrm{~s}^{-1}$ using an ektacytometer based on laser diffraction at $37^{\circ} \mathrm{C}$ (LBY-BX, Precil Company, Beijing, China). For this purpose, a $40-\mu \mathrm{L}$ blood sample was suspended in $1 \mathrm{~mL}$ of a highly viscous solution of polyvinylpyrrolidone ( $\mathrm{PVP}, \mathrm{MW}=30 \mathrm{kDa}$ ) in an isotonic phosphate buffer base (pH 7.4). The EI was obtained based on laser diffraction patterns at user-defined shear stress values.

For the erythrocyte aggregation measurement, the ektacytometer (LBY-BX, Precil Company, Beijing, China) was employed. The measurement was based on the change in back-scattered light on abrupt cessation of the erythrocyte suspension $\left(600\right.$ to $\left.0 \mathrm{~s}^{-1}\right)$ at $37^{\circ} \mathrm{C}[5,21]$. After mixing $500-\mu \mathrm{L}$ blood and $100-\mu \mathrm{L}$ PVP buffer as described above, a $500-\mu \mathrm{L}$ mixture was used to measure the aggregation parameters, including AI and AMP. Hyperaggregation is indicated by increased AI and AMP.

\section{Statistical analysis}

Data are expressed as the mean \pm standard deviation (SD) and were examined for normal distribution and homogeneity of variance using the Shapiro-Wilk test and Levene's test. The means of each group were compared using one-way analysis of variance (ANOVA) followed by the Student-Newman-Keuls test when the normality and homogeneity of variance assumptions were satisfied; otherwise, ANOVA followed by Student-Newman-Keuls multiple range test was applied. $P<0.05$ was considered significant.

\section{Results}

\section{Plasma viscosity}

The plasma viscosity measurements in vitro are presented in Figure 1A. The plasma viscosity was significantly increased in the presence of GEL, HES 130, and HES 200 compared to that of the control group $(P<0.05)$. The plasma viscosity levels were significantly higher in the GEL, HES 130, and HES 200 groups compared to that in the LR group $(P<0.05)$. The plasma viscosity in the HES 200 group was lower than that in the GEL group $(P<0.05)$. Moreover, there was no significant difference between the values in the HES 130 and HES 200 groups.

Plasma viscosity increased significantly after the infusion of GEL $(P<0.05)$ and decreased significantly after the infusion of HES 130, HES 200, and LR compared to the sham group in vivo $(P<0.05$, Figure $1 \mathrm{~B})$. The plasma viscosity levels were significantly higher in the GEL, HES 130, and HES 200 groups compared to that in the LR group $(P<0.05)$. There was no significant difference between the values in the HES 130 and HES 200 groups.

To confirm whether the alterations in plasma viscosity were the result of a direct effect of the intrinsic viscosities of the infused solutions, we performed viscosity measurements of the plasma expanders. The viscosities increased in the following order: LR $<$ GEL $<$ HES $130<$ HES 200 (Table 1).

\section{Erythrocyte deformability}

For the ratio of $3: 1$, the erythrocyte deformability, as measured using EI, significantly decreased in the presence of the four plasma expanders at a shear rate of $100 \mathrm{~s}^{-1}$ $(P<0.05)$. However, the EI values at high rates $\left(>100 \mathrm{~s}^{-1}\right)$ did not differ significantly among the groups (Figure 2A). For the ratio of 5:1, there were no significant differences among the groups at the various shear rates (Figure 2B). In the in vivo study, the EI values at various shear rates for the study groups were not significantly different from those of the sham group (Figure 2C).

\section{Erythrocyte aggregation}

The in vitro erythrocyte aggregation measurements are presented in Figure 3. For the ratio of 3:1, GEL induced hyperaggregation by significantly increasing the $\mathrm{AI}$ and AMP compared to those of the control group. AI was significantly lower in the HES 130 and HES 200 groups compared to that of the GEL group $(P<0.05)$; AMP was significantly lower in the HES 200 group compared to that of the GEL group $(P<0.05)$. There were no significant differences among the HES 130, HES 200, and control groups with respect to AI and AMP $(P>0.05)$; AI was significantly lower in the HES 200 and HES 130 groups compared to that of the LR group $(P<0.05)$. For the ratio of 5:1, GEL induced a dramatic elevation in $\mathrm{AI}$ and AMP compared to those of the control group $(P<0.05)$. The AI and AMP were significantly lower in the HES 200 group compared to those in the GEL group $(P<0.05)$. There were no significant differences among the HES 130, HES 200, and control groups with respect to AI and AMP; AI was significantly lower in the HES 200 and HES 130 groups compared to that in the LR group $(P<0.05$, Figure $3 \mathrm{~A}, \mathrm{~B})$. In addition, there were no differences for AI among the various ratios. The AMP values in the HES 130 and HES 200 groups for the ratio of $3: 1$ were significantly increased compared to those for the ratio of 5:1 $(P<0.05$, Figure 3A, B).

There were no significant differences among the groups with respect to the AI values in vivo (Figure 4A). GEL induced a significant elevation in AMP compared 


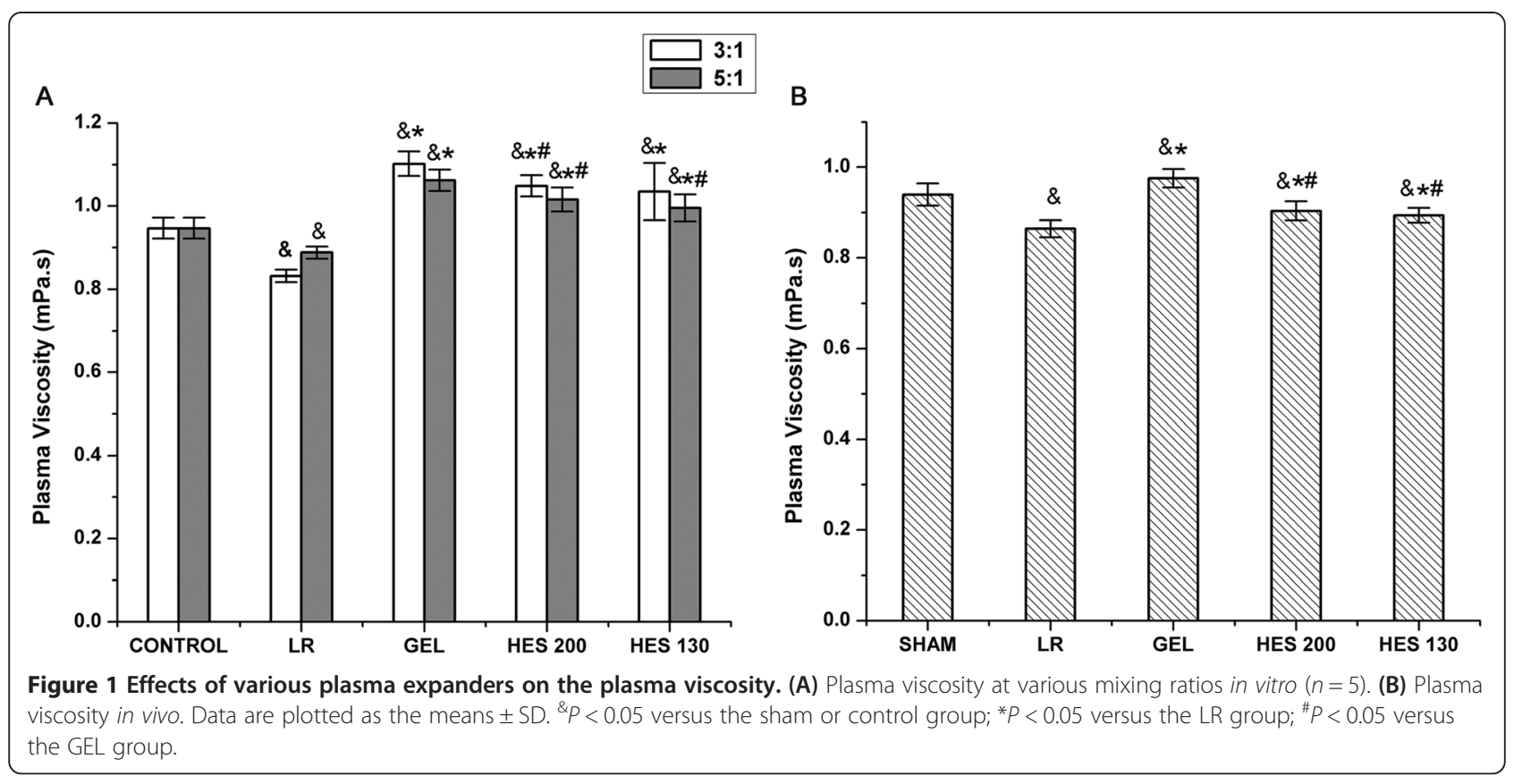

to those of the LR and sham groups $(P<0.05)$. The AMP values in the HES 130 and HES 200 groups were significantly lower than that of the GEL group $(P<0.05)$. There were no significant differences among the HES 130, HES 200, LR, and sham groups with respect to AMP (Figure 4B).

\section{Discussion}

Different fluids under clinical conditions have been found to cause different hemorheological effects, which may contribute to the persistence or deterioration of the physiological state $[7,22]$. The current study was conducted to investigate the influence of synthetic colloids (HES 130, HES 200, and GEL) and LR on plasma viscosity, erythrocyte aggregability, and erythrocyte deformability in vitro and in a rodent model of controlled hemorrhage in vivo.

The hemorheological parameters greatly influence blood circulation [23]. In rat spinotrapezius muscle, Kim et al. demonstrated that induction of erythrocyte aggregation using dextran 500 at a lower arterial pressure reduces the functional capillary density, which is an important indicator of microcirculatory dysfunction [24]. Enhanced erythrocyte aggregation may be associated with decreased wall shear stresses and results in the suppression of nitric oxide release, which plays a key role in the control of circulatory function [25,26]. Furthermore, $\mathrm{O}_{2}$ release from erythrocytes to tissues are physiologically important; erythrocyte aggregation is one of several important factors affecting $\mathrm{O}_{2}$ release in microvessels [27,28]. Restoring the rheological properties of the blood by enhancing the plasma viscosity is beneficial for maintaining the systemic and microhemodynamic parameters during a state of hypovolemic shock [29].

The main finding was the markedly different effects of the buffered and non-buffered solutions on red blood cell aggregation, both in vitro and in a rodent model of controlled hemorrhage in vivo. At the doses used in these experiments, we found that HES 130 and HES 200 did not change erythrocyte aggregation compared to the control. GEL had a potent hyperaggregating effect on rat erythrocyte. Erythrocyte aggregation in the GEL and LR groups was significantly higher compared to those in the HES 130 and HES 200 groups, according to the in vitro study. In the HS model, GEL markedly increased the aggregation amplitude compared to HES 130 and HES 200. However, there was no difference in the aggregation index among the groups. These results are not consistent with previous observations for HES 200 [6,13]. Earlier reports suggested the HES 200 may affect normal erythrocyte rheology. Henkelman et al. demonstrated that HES 200/0.5 enhanced erythrocyte aggregation in vitro using a

Table 1 Intrinsic viscosity of the four plasma expanders

\begin{tabular}{lllll}
\hline & LR & GEL & HES 200 & HES 130 \\
\hline Intrinsic viscosity (mPa.s) & $0.673 \pm 0.002$ & $1.441 \pm 0.015^{*}$ & $1.797 \pm 0.013^{\# *}$ & $1.529 \pm 0.015^{\# * \S}$ \\
\hline
\end{tabular}

Values are means \pm SD.

${ }^{*} P<0.05$ versus $\mathrm{LR},{ }^{\S} P<0.05$ versus HES $200,{ }^{\#} P<0.05$ versus GEL. 


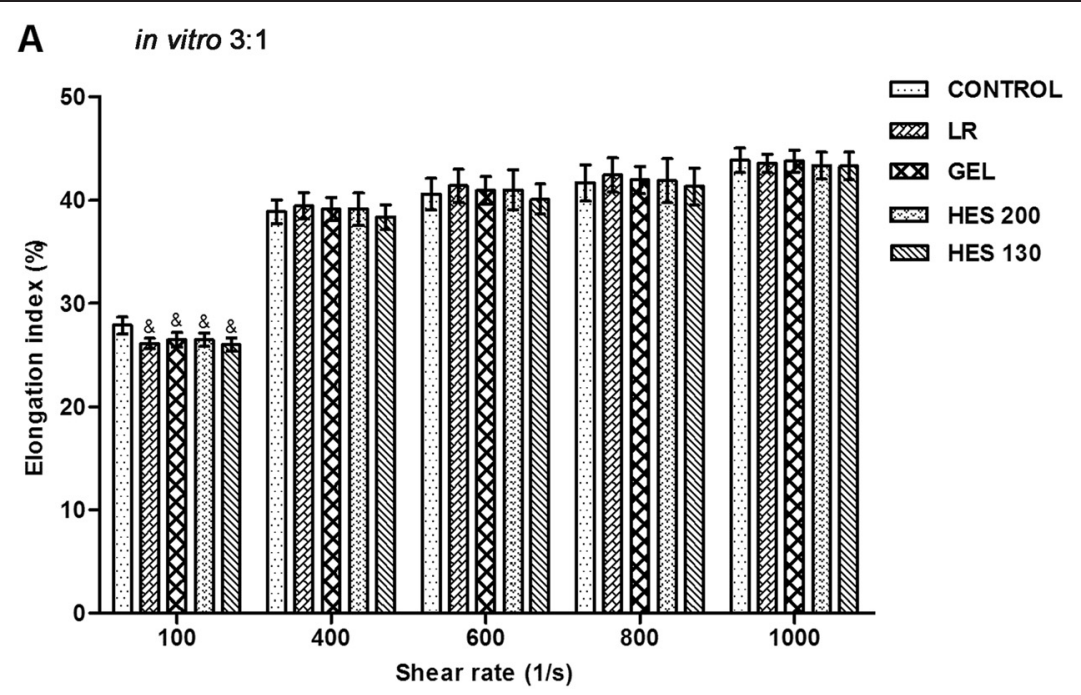

B in vitro $5: 1$
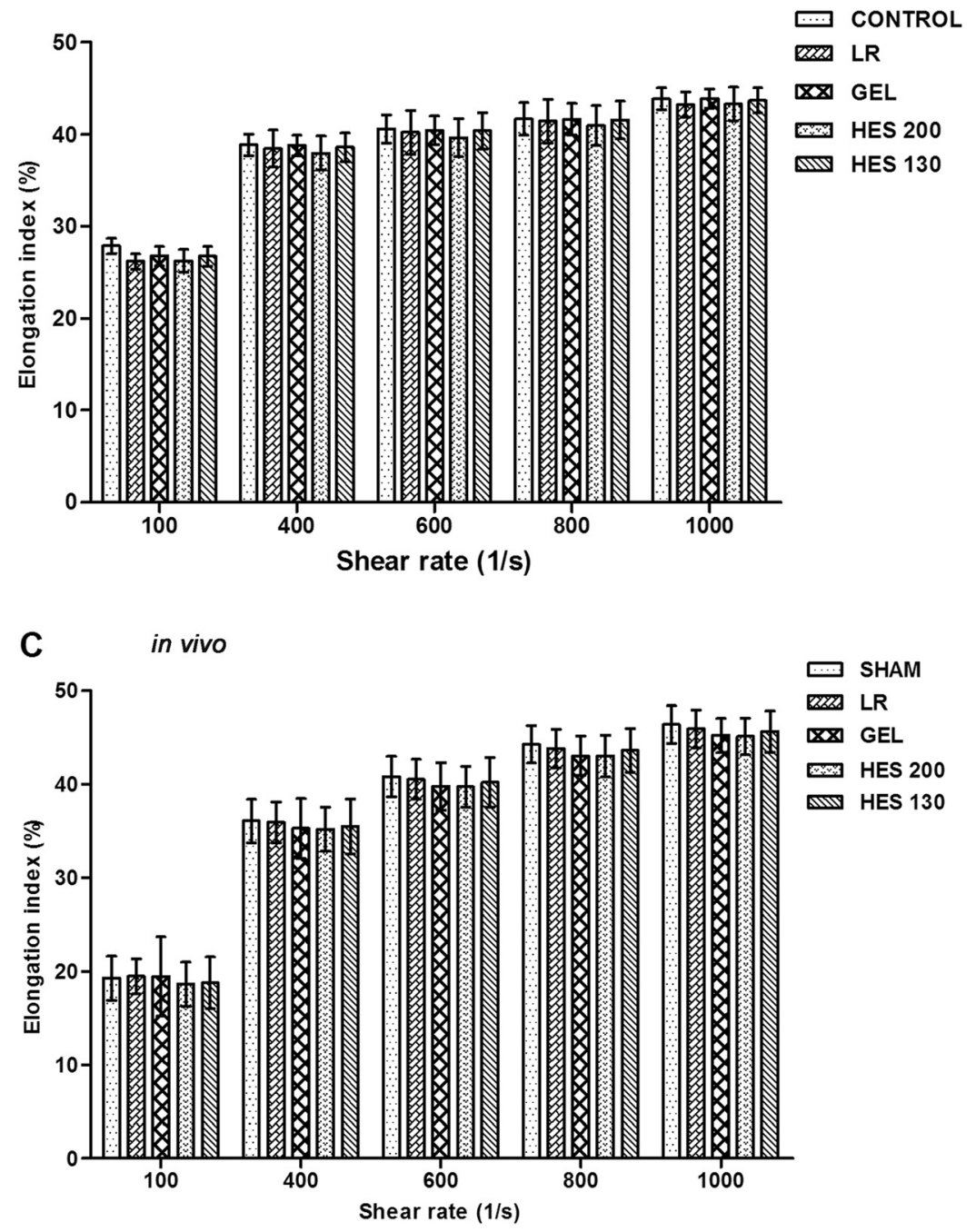

Figure 2 Effects of various plasma expanders on erythrocyte deformability. (A) Erythrocyte deformability at a ratio of 3:1 in vitro ( $n=5$ ). (B) Erythrocyte deformability at a ratio of 5:1 in vitro $(n=5)$. (C) Erythrocyte deformability in vivo. Data are plotted as the means \pm SD. ${ }^{\&} P<0.05$ versus the control group. 


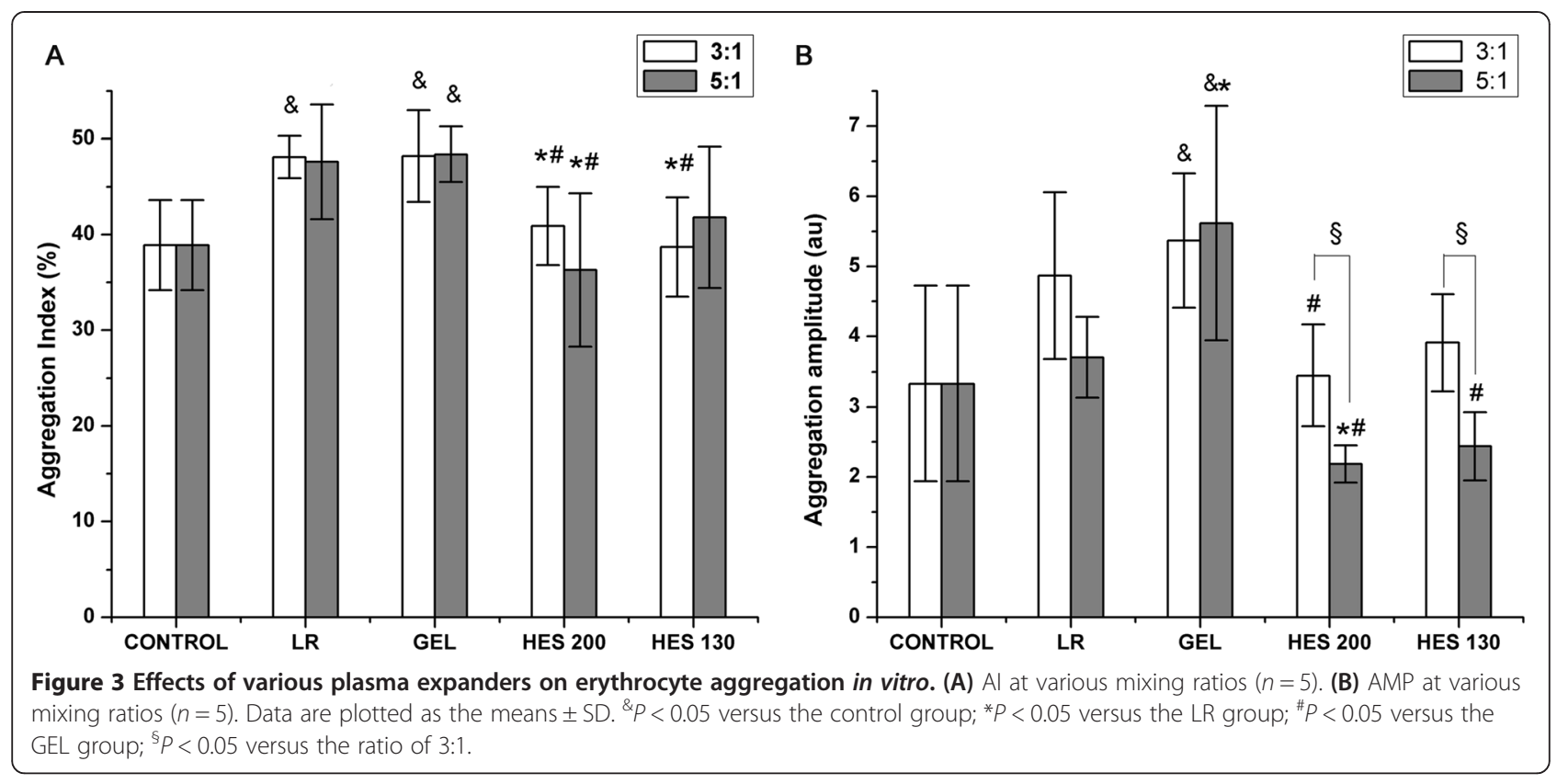

laser-assisted optical rotational cell analyzer [14]. Differences in the concentration of colloid may account for these controversial results. In this study, erythrocyte aggregation was tested on erythrocytes suspended in $10 \%$ 200-kDa HES solutions. The final concentration of HES is higher than that of our present study (1\% and 1.5\%). It has been shown that the aggregation of erythrocytes was extended and accelerated by increasing the concentration of HES [13]. In contrast, our results for HES were consistent with a report of preoperative hemodilution [4]. The erythrocyte aggregation was unchanged with HES 200 in the study.
In this study, the ability of erythrocytes to form aggregates was measured by the aggregation amplitude and aggregation index. The prominent effect observed in vitro is in accordance with in vivo data for aggregation amplitude. However, the data for aggregation index in the in vitro study is not in accordance with the in vivo data. Our results revealed this incongruence between the in vivo and in vitro studies. The aggregation amplitude represents the extent of the aggregation. The overall aggregation behavior of the suspension is described by the aggregation index. The aggregation amplitude may be more sensitive than the aggregation index. It has

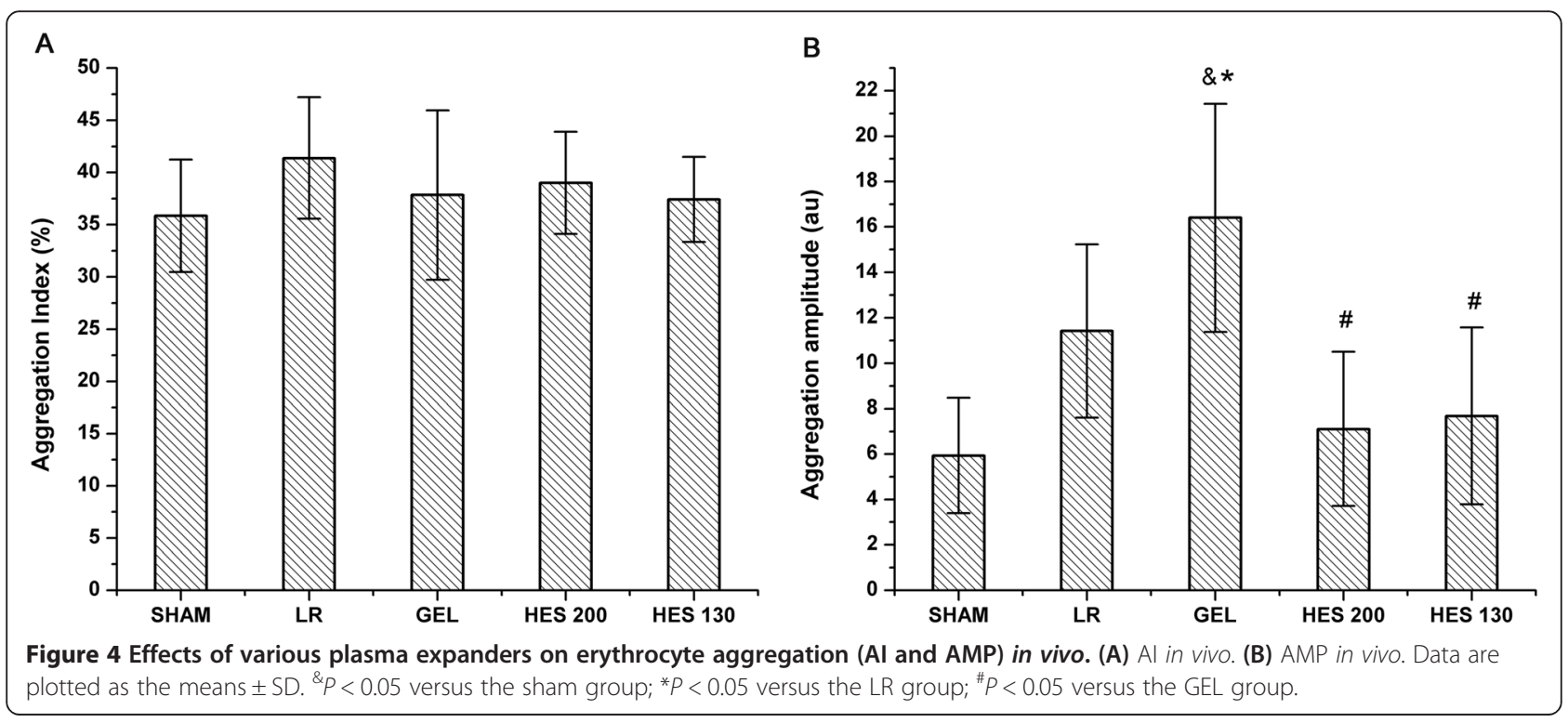


been indicated that the aggregation amplitude is significantly higher in patients with slow coronary flow, whereas there was no significant difference between the groups with respect to the aggregation index [30]. In another study, Hazer et al. demonstrated the effect of pravastatin on hemorheological parameters in a rodent traumatic brain injury model. The aggregation amplitude first increased from the second day to the seventh day in the sham-operated group; however, the aggregation index remained stable on the seventh day. In the pravastatin group, the aggregation amplitude significantly increased on the 15th day, whereas the aggregation index remained stable on the 15th day [31]. This finding indicates that the aggregation amplitude should be taken into consideration when evaluating the erythrocyte aggregation, especially during the early stages of changes in the erythrocyte aggregation.

There have been few studies on colloid concentrations in plasma after hemorrhagic shock/resuscitation. The final concentrations (1\% and 1.5\%) for HES that were used in the present in vitro study were comparable to values observed in clinical studies. It was reported that patients undergoing orthopedic surgery have mean HES plasma concentrations of approximately $1 \%$ at the end of surgery [17]. During hemodilution therapy in patients with cerebrovascular diseases, the mean HES plasma peak concentration was $1.5 \%$ [18]. Therefore, the in vitro data we present are likely to reflect clinical conditions during large-dose colloid administration.

The plasma viscosity was significantly increased when GEL was used compared with HES 130 and HES 200, both in vitro and in vivo. However, the intrinsic viscosity of GEL was lower than those of HES 130 and HES 200. The various interactions between colloids and plasma proteins may account for this result. Previous studies have indicated that the solution viscosity increases with the rise of molecular mass of a given type of colloid at a constant concentration [32,33]. As mentioned above, gelatin has also been shown to interact specifically with plasma proteins, such as fibronectin [34], and may therefore enhance the formation of macromolecules, leading to increased plasma viscosity.

In our previous study, we compared the effects of different solutions including HES 130, HES 200, GEL, LR, normal saline, and Ringer acetate on hemorheology in an in vitro study and found there were no significant differences for hemorheological parameters between isotonic crystalloids. Therefore, we finally chose LR as the control in this study. In addition, the results in vitro are not directly comparable with in vivo coagulation in rats.

For the ratio of 3:1 in vitro study, LR was indicated to increase AI. There is no doubt that LR can decrease plasma protein concentrations and plasma viscosity. The decrease of plasma protein concentrations results in the inhibition of erythrocyte aggregation [35,36]. The result in this study implies that other factors also were involved in the increased erythrocyte aggregation. The divalent cations $\left(\mathrm{Ca}^{+}\right)$contained in LR are indicated to exacerbate the erythrocyte aggregation by decreasing the erythrocyte surface potential and electric double layer [37]. In addition, although it remains controversial, the lactate level was positively correlated with erythrocyte aggregation [38,39]. Consequently, the divalent cations and lactate may account for the increased erythrocyte aggregation.

A previous study has indicated that the $\mathrm{pH}$ levels can affect the erythrocyte aggregation [40]. There is a huge range from four different solutions (4.7-7.1). However, we found that the $\mathrm{pH}$ level of blood samples showed negligible changes when the samples mixed with four different solutions. It is inferred that the buffering capacity of the blood samples may prevent the $\mathrm{pH}$ levels from changing. The $\mathrm{pH}$ levels did not account for the difference of erythrocyte aggregation in this study.

The present study has limitations. First, the present in vivo study examined only a single time point, i.e., $2 \mathrm{~h}$ after treatment. Second, the results of blood gas and lactate concentration were absent. In addition, the volume of resuscitation was three times that of the blood withdrawal for LR in this study. The volume may hyperinfuse the rats, which may have an impact on the results.

\section{Conclusions}

In summary, our study indicates that different synthetic colloids used for resuscitation produce various hemorheological effects, particularly with respect to erythrocyte aggregation. We also determined that GEL significantly accelerates the erythrocyte aggregation and significantly elevates the plasma viscosity compared to hydroxyethyl starch; furthermore, hydroxyethyl starch did not change the erythrocyte aggregation compared to that of the control group. In addition, in vitro hemorheological measurements are likely to provide hints for in vivo studies.

\section{Competing interests}

The authors declare that they have no competing interests.

\section{Author's contributions}

GC conceived the study, carried out the experiments, and wrote the manuscript. JZ and PL performed the surgical procedures and helped measure the plasma viscosity. XK, GY, YW, and YY helped draft the manuscript and helped measure the erythrocyte aggregability and deformability. $X \mathrm{~L}$ and $Y Z$ contributed to the statistical analyses. $\mathrm{LZ}$ and $\mathrm{HZ}$ are the Principal Investigators and take responsibility for all conceptual and technical aspects of this study. All authors read and approved the final manuscript.

\section{Acknowledgements}

This work was supported by grants from the National Natural Science Foundation of China (No. 31271001) and the National High Technology Research and Development Program of China (No.2012AA021902). 
Received: 20 July 2014 Accepted: 19 January 2015

Published online: 04 February 2015

\section{References}

1. Bagshaw SM, Bellomo R. The influence of volume management on outcome. Curr Opin Crit Care. 2007;13:541-8.

2. Standl T, Burmeister MA, Schroeder F, Currlin E, Schulte am Esch J, Freitag M, et al. hydroxyethyl starch (HES) 130/0.4 provides larger and faster increases in tissue oxygen tension in comparison with prehemodilution values than HES 70/0.5 or HES 200/0.5 in volunteers undergoing acute normovolemic hemodilution. Anesth Analg. 2003;96:936-43.

3. Neff TA, Fischler L, Mark M, Stocker R, Reinhart WH. The influence of two different hydroxyethyl starch solutions (6\% HES 130/0.4 and 200/0.5) on blood viscosity. Anesth Analg. 2005;100:1773-80.

4. Audibert $\mathrm{G}$, Dormer M, Lefèvre JC, Stoltz JF, Laxenaire MC. Rheologic effects of plasma substitutes used for preoperative hemodilution. Anesth Analg. 1994:78:740-5.

5. Freyburger G, Dubreuil M, Boisseau MR, Janvier G. Rheological properties of commonly used plasma substitutes during preoperative normovolaemic acute haemodilution. Br J Anaesth. 1996;76:519-25.

6. Castro VJ, Astiz ME, Rackow EC. Effect of crystalloid and colloid solutions on blood rheology in sepsis. Shock. 1997:8:104-7.

7. Zhao L, Wang B, You GX, Wang ZL, Zhou H. Effects of different resuscitation fluids on the rheologic behavior of red blood cells, blood viscosity and plasma viscosity in experimental hemorrhagic shock. Resuscitation. 2009;80:253-8.

8. Pribush A, Zilberman-Kravits D, Meyerstein N. The mechanism of the dextran-induced red blood cell aggregation. Eur Biophys J. 2007;36:85-94.

9. Kroemer $\mathrm{H}$, Haass A, Muller K, Jager H, Wagner EM, Heimburg P, et al. Haemodilution therapy in ischaemic stroke: plasma concentrations and plasma viscosity during long-term infusion of dextran 40 or hydroxyethyl starch 200/0.5. Eur J Clin Pharmacol. 1987;31:705-10.

10. Sümpelmann R, Günther A, Zander R. Haemoconcentration by gelatin-induced acceleration of erythrocyte sedimentation rate. Anaesthesia. 2000;55:217-20.

11. Vicaut E. Opposite effects of red blood cell aggregation on resistance to blood flow. J Cardiovasc Surg (Torino). 1995;36:361-8.

12. Yedgar $\mathrm{S}$, Koshkaryev A, Barshtein $\mathrm{G}$. The red blood cell in vascular occlusion. Pathophysiol Haemost and Thromb. 2002;32:263-8.

13. Morariu AM, Vd Plaats A, V Oeveren W, T Hart NA, Leuvenink HG, Graaff R, et al. Hyperaggregating effect of hydroxyethyl starch components and University of Wisconsin solution on human red blood cells: a risk of impaired graft perfusion in organ procurement? Transplantation. 2003;76:37-43.

14. Henkelman S, Rakhorst G, van der Mei HC, Busscher HJ. Use of hydroxyethyl starch for inducing red blood cell aggregation. Clin Hemorheol Microcirc. 2012;52:27-35.

15. Ehrly AM, Seebens H, Saeger-Lorenz K. [Effect of a 10\% and $6 \%$ hydroxyethyl starch solution (molecular weight 200,000/0.62) in comparison with a $10 \%$ dextran solution (molecular weight 40,000) on flow properties of blood and tissue oxygen pressure in patients with intermittent claudication]. Infusionstherapie. 1988;15:181-7.

16. Menu P, Bleeker W, Longrois D, Caron A, Faivre-Fiorina B, Muller S, et al. In vivo effects of $\mathrm{Hb}$ solutions on blood viscosity and rheologic behavior of RBCs: comparison with clinically used volume expanders. Transfusion. 2000;40:1095-103.

17. Jungheinrich C, Sauermann W, Bepperling F, Vogt NH. Volume efficacy and reduced influence on measures of coagulation using hydroxyethyl starch 130/0.4 (6\%) with an optimised in vivo molecular weight in orthopaedic surgery : a randomised, double-blind study. Drugs R D. 2004;5:1-9.

18. Treib J, Haass A, Pindur G, Treib W, Wenzel E, Schimrigk K. Influence of intravascular molecular weight of hydroxyethyl starch on platelets. Eur J Haematol. 1996:56:168-72.

19. Baskurt OK, Boynard M, Cokelet GC, Connes P, Cooke BM, Forconi S, et al. New guidelines for hemorheological laboratory techniques. Clin Hemorheol Microcirc. 2009;42:75-97.

20. Chen G, You G, Wang Y, Lu M, Cheng W, Yang J, et al. Effects of synthetic colloids on oxidative stress and inflammatory response in hemorrhagic shock: comparison of hydroxyethyl starch 130/0.4, hydroxyethyl starch 200/0.5, and succinylated gelatin. Crit Care. 2013;17:R141.

21. Hardeman MR, Dobbe JGG, Ince C. The Laser-assisted Optical Rotational Cell Analyzer (LORCA) as red blood cell aggregometer. Clin Hemorheol Microcirc. 2001;25:1-11.
22. Chen G, Zhao L, Liu Y, Liao F, Han D, Zhou H. Regulation of blood viscosity in disease prevention and treatment. Chin Sci Bull. 2012;57:1946-52.

23. Nolte D, Botzlar A, Pickelmann S, Bouskela E, Messmer K. Effects of diaspirin-cross-linked hemoglobin (DCLHb) on the microcirculation of striated skin muscle in the hamster: a study on safety and toxicity. J Lab Clin Med. 1997;130:314-27.

24. Kim S, Popel AS, Intaglietta M, Johnson PC. Effect of erythrocyte aggregation at normal human levels on functional capillary density in rat spinotrapezius muscle. Am J Physiol Heart Circ Physiol. 2006;290:H941-7.

25. Forconi S, Gori T. Endothelium and hemorheology. Clin Hemorheol Microcirc. 2013;53:3-10.

26. Baskurt OK, Yalcin O, Ozdem S, Armstrong JK, Meiselman HJ. Modulation of endothelial nitric oxide synthase expression by red blood cell aggregation. Am J Physiol Heart Circ Physiol. 2004;286:H222-9.

27. Tateishi N, Suzuki Y, Cicha I, Maeda N. O2 release from erythrocytes flowing in a narrow O2-permeable tube: effects of erythrocyte aggregation. Am J Physiol Heart Circ Physiol. 2001;281:H448-56.

28. Tateishi N, Suzuki Y, Shirai M, Cicha I, Maeda N. Reduced oxygen release from erythrocytes by the acceleration-induced flow shift, observed in an oxygen-permeable narrow tube. J Biomech. 2002;35:1241-51.

29. Cabrales P, Tsai AG, Intaglietta M. Increased plasma viscosity prolongs microhemodynamic conditions during small volume resuscitation from hemorrhagic shock. Resuscitation. 2008;77:379-86.

30. Ergun-Cagli K, lleri-Gurel E, Ozeke O, Seringec N, Yalcinkaya A, Kocabeyoglu $\mathrm{S}$, et al. Blood viscosity changes in slow coronary flow patients. Clin Hemorheol Microcirc. 2011;47:27-35.

31. Hazer DB, Berker M, Narin F, lleri-Gurel E, Basak AT, Seringec N, et al. Effects of pravastatin on cellular ultrastructure and hemorheology in rats after traumatic head injury. Clin Hemorheol Microcirc. 2010;46:1-11.

32. Neu B, Wenby R, Meiselman HJ. Effects of dextran molecular weight on red blood cell aggregation. Biophys J. 2008;95:3059-65.

33. Li X, Xia W. Effects of concentration, degree of deacetylation and molecular weight on emulsifying properties of chitosan. Int J Biol Macromol. 2011;48:768-72.

34. Balian G, Click EM, Crouch E, Davidson JM, Bornstein P. Isolation of a collagen-binding fragment from fibronectin and cold-insoluble globulin. J Biol Chem. 1979;254:1429-32.

35. Wells RE, Gawronski TH, Cox PJ, Perera RD. Influence of fibrinogen on flow properties of erythrocyte suspensions. Am J Physiol. 1964;207:1035-40.

36. Baskurt OK, Temiz A, Meiselman HJ. Red blood cell aggregation in experimental sepsis. J Lab Clin Med. 1997;130:183-90.

37. Jan KM, Chien S. Influence of the ionic composition of fluid medium on red cell aggregation. J Gen Physiol. 1973;61:655-68.

38. Brun JF, Supparo I, Fons C, El Bouhmadi A, Orsetti A. Low values of blood viscosity and erythrocyte aggregation are associated with lower increases in blood lactate during submaximal exercise. Clin Hemorheol Microcirc. 1994;14:105-16.

39. Brun JF, Belhabas H, Granat MC, Sagnes C, Thöni G, Micallef JP, et al. Postexercise red cell aggregation is negatively correlated with blood lactate rate of disappearance. Clin Hemorheol Microcirc. 2002;26:231-9.

40. Cicha I, Suzuki Y, Tateishi N, Maeda N. Changes of RBC aggregation in oxygenation-deoxygenation: $\mathrm{pH}$ dependency and cell morphology. Am J Physiol. 2003;284:H2335-42.

\section{Submit your next manuscript to BioMed Central and take full advantage of:}

- Convenient online submission

- Thorough peer review

- No space constraints or color figure charges

- Immediate publication on acceptance

- Inclusion in PubMed, CAS, Scopus and Google Scholar

- Research which is freely available for redistribution

Submit your manuscript at www.biomedcentral.com/submit

C) Biomed Central 